\title{
BIENESTAR Y LEGITIMIDAD
}

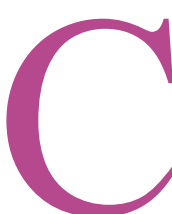

omo es sabido, el término «bienestar» ha sido utilizado en textos constitucionales recientes y también del pasado en vinculación con tareas que incumben al Estado y cuya importancia es considerada tan grande que su no cumplimiento podría poner en duda la legitimidad misma del régimen político. Así, por ejemplo, la actual Constitución Española establece en su artículo 50, al hacer referencia a la tercera edad, que los «poderes públicos... promoverán su bienestar mediante un sistema de servicios sociales...» También en el artículo 31 de la Constitución de la Confederación Suiza se afirma que la Confederación «tornará las medidas adecuadas para aumentar el bienestar general y procurar la seguridad económica de sus ciudadanos».

La noción de bienestar parece estar relacionada en este contexto con aquellas condiciones que son necesarias para llevar una vida digna y que el Estado debería promover o asegurar. Se trataría no sólo del deber del Estado de garantizar la satisfacción de las necesidades primarias de la población, sino también de tomar las medidas pertinentes con el fin de elevar su calidad de vida.

En el Preámbulo de la Constitución de los Estados Unidos de América, por otra parte, se incluye entre los fines esenciales de esa Constitución el de «promover el bienestar general y asegurar... los beneficios de la libertad». Y en una noble Constitución española, la liberal de 1812, se establece como fin del gobierno la promoción del bienestar, entendido también como felicidad de los ciudadanos. En efecto, su artículo 13 reza: «El objeto del gobierno es la felicidad de la Nación, puesto que el fin de toda sociedad política no es otro que el bienestar de los individuos que la componen.»

El concepto de bienestar parece estar vinculado aquí con la idea de felicidad. La importancia de la felicidad (¿bienestar?) fue subrayada por Thomas Jefferson en su proyecto de Declaración 
de Independencia de los Estados Unidos. Como se recordará, en este texto, Jefferson sostenía que entre las verdades sagradas e innegables figuraban que «todos los hombres son creados iguales e independientes; que de la igual creación ellos derivan derechos inherentes e inalienables, entre los cuales están la preservación de la vida y de la libertad y la persecución de la felicidad; para asegurar estos fines son instituidos los gobiernos entre los hombres».

Jefferson seguía aquí la concepción de Burlamaqui según la cual todos los hombres tenían el deber de preservar la propia vida, de perfeccionar su razón y de perseguir su propia felicidad; y por tener este deber tenían también derecho a la satisfacción de estos tres principios ${ }^{1}$.

Ya la mención de estos textos constitucionales y declaraciones básicas permite inferir la importancia central del concepto de bienestar pero, al mismo tiempo, pone de manifiesto la vaguedad y ambigüedad de este concepto. En efecto, mientras en algunos pasajes (artículo 50 de la Constitución Española) el bienestar parece estar más vinculado con lo que suele llamarse niveles de vida o calidades de vida, en otros como en la Constitución Española de 1812 y en el documento de Jefferson, hace referencia a la felicidad entendida como un estado mental subjetivo.

He traído a colación estos textos porque creo que ellos ponen de manifiesto algunas dificultades vinculadas con la definición del bienestar. Mientras por una parte se apunta más bien a notas objetivas de cómo ha de ser el mundo, en otras se apunta mas bien a aspectos subjetivos. También la bibliografía actual del concepto de bienestar escoge estas dos orientaciones, y los autores suelen oscilar entre posiciones objetivistas y subjetivistas.

No he de entrar aquí a analizar las diferentes versiones que se han dado de bienestar, sino que me interesa vincular dos concepciones básicas: la que aproxima el bienestar a la felicidad individual y la que apunta más bien hacia condiciones objetivas del mundo. Y esta consideración quiero realizarla desde la perspectiva de las consecuencias que tiene una y otra versión con respecto al problema de la legitimidad de un régimen político. A tal fin habré de examinar: I el concepto de bienestar entendido como felicidad, II el concepto de bienestar entendido como nivel de vida, considerando igualmente qué papel debe jugar el Estado

${ }^{1}$ Las citas de Jefferson y Burlamaqui en M. White, The Philosophy of the American Revolution, Oxford University Press, 1981, pág. 162. 
en el primer caso y cuál en el segundo. Finalmente, III, habré de formular algunas consideraciones acerca de la importancia del concepto de bienestar entendido en una u otra forma en relación con uno de los principios básicos del Estado democrático de tradición liberal; es decir: el respeto de las personas en tanto seres autónomos.

Si se entiende el bienestar como felicidad, hay que tener siempre en cuenta que la felicidad es un estado de la mente; es un estado subjetivo que está estrechamente vinculado en la satisfacción de los deseos. Como sostiene Ted Honderich, la felicidad es precisamente lo opuesto a la desgracia. Mientras la felicidad constituye el logro de los deseos y puede, por ello, ser considerada en términos de satisfacción, la desgracia está relacionada con los deseos no cumplidos y, por tanto, más vinculada con la idea de frustración ${ }^{2}$.

Naturalmente, las personas pueden tener una gran variedad de deseos, según sean los valores que ellas mismas posean. Siempre que yo deseo algo, lo hago en virtud de que ese algo es valioso para mí. Mi felicidad radica en alcanzar mis propios objetivos, valores, preferencias, etc. Entendidos de esta manera, los deseos reciben el nombre de «actuales» o «reales».

La pregunta es: ¿debe el estado promover y garantizar -tal como rezan los distintos textos constitucionales- la felicidad de los ciudadanos entendida como satisfacción de los deseos actuales? La respuesta no puede ser sino negativa. Y no solamente debido a la escasez moderada de recursos existentes que hace prácticamente imposible la satisfacción de todos y cada uno de los deseos posibles de los ciudadanos; sino también porque dentro de un contexto poblacional donde reinan los grandes números resulta difícil conocer con exactitud la totalidad de los deseos actuales de todas las personas y la intensidad con que ellos se manifiestan. Pero existe aún un problema más grave.

Los seres humanos tienen deseos muy de diversa índole. Mientras poseen algunos cuyo contenido es manifiestamente altruista y cuya satisfacción favorece la cooperación social, suelen tener otros que se caracterizan por ser antisociales o inmorales. Que en estos casos el Estado deba promover y garantizar la satisfacción

${ }^{2}$ Cfr. Ted Honderich, «The Question of Well-being and the Principle of Equality», en Mind, vol. XC, 1981, págs. 487 y sigs. 
de tales deseos para favorecer la felicidad de sus agentes no deja de ser una pretensión moralmente perversa y absolutamente rechazable.

Es por esa razón que ni el artículo 13 de la Constitución de Cádiz antes mencionada, ni la Declaración de Jefferson podrían ser interpretados en el sentido de que es deber del estado promover y asegurar la persecución de la felicidad de sus ciudadanos, entendida ésta como la satisfacción de sus deseos actuales.

Los inconvenientes que presenta la versión de considerar la felicidad como la satisfacción de los deseos actuales de las personas ha llevado a numerosos autores a formular ciertas restricciones o cualificaciones sobre esos deseos con la finalidad de evitar que se produzcan tales despropósitos. Hare, por ejemplo, habla de «preferencias absolutamente prudentes»; Harsayni quiere excluir todas las «preferencias antisociales como el sadismo, la envidia, el resentimiento y el encono». Y en un trabajo reciente, James Griffin ha propuesto que los deseos sean «racionales» o «informados».

Los deseos son «Informados» si los agentes que los poseen aprecian la verdadera naturaleza de los objetivos que se proponen. Los deseos informados tendrían la propiedad de evitar los defectos que acusa un agente que sólo persigue la satisfacción de sus deseos actuales y que son motivados, por ejemplo, por carecer de una información completa, escoger medios inadecuados para alcanzar los fines perseguidos, desconocer la red conceptual que le permita afrontar los diferentes problemas prácticos con probabilidades de éxito, etc. En definitiva, un deseo es «informado» si se construye sobre la base de la correcta apreciación de la verdadera naturaleza de sus objetivos, incluyendo cualquier cosa necesaria para alcanzarlo ${ }^{3}$.

La versión de los deseos informados trata de responder a la pregunta acerca de qué cosas debería escoger una persona, más que el hecho empírico de interrogarse acerca de qué cosas desea efectivamente esa persona. Desde esta nueva perspectiva, la felicidad consistiría en el logro de todos aquellos deseos informados que, con independencia de nuestras servidumbres individuales y circunstancias contingentes que nos rodean, todos los seres humanos deberíamos tener.

El propio Griffin, sin embargo, se encarga de mostrar las dificultades que tiene que asumir este tipo de deseos cuya satisfacción puede ser identificada con la felicidad y, por tanto, como

${ }^{3}$ Cfr. James Griffin, Well-being. Its meaning, measuremet and moral importance, Oxford, Clarendon Press, 1986, pág. 14. 
una versión del bienestar. En efecto, si los deseos a satisfacer no cambian jamás (por ser «informados») entonces se podría hacer un orden lexicográfico en cuanto a su satisfacción. Pero la vida no es tan simple, las situaciones en las cuales se ve inmersa una persona cambian, sus preferencias se modifican y no siempre en una única dirección. Por otra parte, al ampliar tanto la variedad de deseos que deberíamos satisfacer -recuérdese que son «informados»; es decir: deberían formularse con independencia de si actualmente se poseense podrían desear cosas que para nada afectan el propio plan de vida y, por consiguiente, resultan irrelevantes para el bienestar ${ }^{4}$.

Pero el esfuerzo por circunscribir el bienestar de las personas a su felicidad, entendida como la satisfacción de sus deseos en cualquiera de sus dos versiones (actuales o informados) pronto se manifiesta insuficiente. Ya que la satisfacción de los deseos conduce a la felicidad y ésta es un estado mental subjetivo, resultará que se puede lograr por muchos y variados caminos. Según Amartya Sen, los deseos reflejan compromisos con la realidad y la realidad es para algunos más dura que para otros. En su opinión, un desgraciado indigente que solamente desea sobrevivir o un ama de casa sometida que lucha por lograr su individualidad pueden haber aprendido a contener sus deseos con el objeto de salvar su difícil situación. Pero, naturalmente, parecería un poco grotesco identificar la satisfacción de esos la satisfacción de esos pocos y limitados deseos con el bienestar de esas personas ${ }^{5}$. No resulta extraño, pues, que en más de una ocasión se haya puesto en duda la fertilidad de la idea de equiparar la satisfacción de deseos con la noción de bienestar.

Por otra parte, las fuentes que regulan la producción de los deseos de una persona son de la más variada índole. Y la motivación que alegan estas personas para alcanzar sus logros y para la realización de sus acciones exceden con creces la persecución de su propio bienestar. En casos como esos, no está claro cuales son las ventajas de centrar el bienestar de una persona en la satisfacción de sus deseos.

Existen todavía dos argumentos más en contra de esa pretensión. El primero, es que no siempre las personas persiguen la maximización de la satisfacción de sus deseos, los individuos tienen a menudo deseos moderados y se contentan con haber logrado una satisfacción tan solo parcial, sin que ello implique

${ }^{4}$ Idem, págs. 16 y 17.

${ }^{5}$ Cfr, Amartya Sen, «The Standard of Living», en The Tanner Lectures on Human Values, Cambridge University Press, 1986, págs. 16 y 17. 
decir con propiedad que su bienestar sea moderado o parcial ${ }^{6}$. El segundo, es que las personas pueden actualmente carecer del interés de alcanzar alguna cosa, y no precisamente en virtud de que la considere disvaliosa o indigna de ser perseguida, sino porque, dadas las circunstancias que rodean al caso, ha perdido las esperanzas de lograr su satisfacción. Y como resulta obvio, la mengua de algunos de los elementos que constituyen la clase de los deseos de una persona no significa, estrictamente hablando, una mengua de su bienestar.

Además, según Sen, identificar la felicidad con el bienestar tiene el inconveniente no sólo que hace ignorar otros aspectos muy importantes para el bienestar de las personas -como sería alguien que viviendo en condiciones infrahumanas se sintiera «feliz» a causa de su consumo de opio- sino también porque hay estados mentales diferentes de la felicidad, como la excitación, por ejemplo, que tienen una importancia especial para la consideración del bienestar de una persona. Por estas razones, agrega Sen, «es difícil evitar la conclusión que aunque la felicidad tiene una obvia y directa relevancia para el bienestar, resulta inadecuada como representación del mismo» ${ }^{7}$.

Pero aún cuando se aceptara que el bienestar equivale a la satisfacción de los deseos de las personas quedaría todavía por responder qué actitud debería adoptar el Estado. El Estado, repito, carece de las capacidades y de los medios materiales para conocer cuales son todos los deseos -actuales o informados- de las personas. Y se corre el riesgo de que debido a ese desconocimiento, o a causa de tratar de compatibilizar los deseos de unos que entran en conflicto flagrante con los de los otros, o simplemente en virtud de que considere que los deseos que valora son objetivamente mejores -o suficientemente informados-y que, en consecuencia constituyen aquellos intereses que más conviene a la Nación, trate de imponer sus propios criterios de felicidad, incurriendo en un perfeccionismo moral éticamente injustificado. Sobre este punto volveré más adelante.

Si los intentos de mostrar el bienestar como un estado subjetivo de la mente se han manifestado insuficientes, habrá que seguir una vía de análisis diferente. Esta vez basada en criterios objetivos. Por esa razón pasaré a considerar a continuación el concepto de bienestar como nivel de vida.

${ }^{6}$ Idem, págs. 17 y sigs.

${ }^{7}$ Cfr. Amartya Sen, «Well-being and Freedom», en The Journal of Philosophy, vol. LXXXII, núm. 4, abril de 1985, págs. 188 y 189. 
El concepto de bienestar como nivel de vida hace referencia a cuestiones objetivas. Está relacionado al estado del mundo; es decir, a cómo es el mundo en realidad.

En general, se subraya que para juzgar el nivel de vida de una persona se ha de tomar en consideración un buen número de variables. Se ha de conocer, por ejemplo, cuál es el conjunto de sus necesidades básicas, qué cantidades de bienes de consumo posee y cuáles son sus ingresos periódicos. Se ha de tener información, además, de una serie de datos que no siempre son transferibles de una persona a otra. Saber cuál es el entorno familiar de una persona, cuáles son sus capacidades y habilidades para desarrollar una actividad productiva, cuáles son los niveles de salud propio y los de sus allegados, o la posibilidad de disponer de tiempo para su ocio, constituyen entre muchas otras cosas más, cuestiones absolutamente relevantes.

No creo necesario subrayar el hecho de que no existe ningún listado completo que permita definir exactamente lo que es el nivel de vida de una persona. Como tampoco existe ningún criterio objetivo que permita la comparación que haga posible decidir cuál de dos personas goza de una mayor calidad de vida: si aquélla que dispone de una buena salud y de un entorno familiar afectivo y acorde a sus aspiraciones, por ejemplo, o aquella otra que tiene una posición económica acomodada, dispone de tiempo libre para su ocio pero padece una cruel enfermedad. A pesar de estos inconvenientes, se han utilizado criterios cuya aceptación está más o menos generalizada y que permite fijar, siempre dentro de una cierta convencionalidad, qué debe considerarse como nivel o calidad de vida y cómo han de utilizarse para que puedan ser posibles las comparaciones interpersonales.

A los efectos de este trabajo y en aras de la simplificación del problema consideraré, siguiendo a Ted Honderich ${ }^{8}$, que el nivel de vida de una persona puede ser fijado por la presencia o ausencia de estas seis categorías: subsistencia, bienes materiales no imprescindibles, respeto, libertad, relaciones personales y cultura.

1. No está en discusión que el primer elemento a considerar para la determinación del nivel de vida de una persona está relacionado

${ }^{8}$ Cfr. Ted Honderich, obra citada, págs. 481 y sigs. 
con el aseguramiento de sus necesidades básicas, de su subsistencia, la de su pareja y la de sus hijos. Se trata de gozar de un mínimo de condiciones objetivas que permitan el desarrollo de la propia vida y que tienen que ver con un mínimo de alimentación, de salud, de vivienda, etc. La satisfacción de esta categoría es básica y tiene absoluta prioridad sobre las demás, cosa que no ocurre entre las restantes cinco categorías.

2. La segunda categoría hace referencia a la posesión de aquellos bienes que no son imprescindibles como fines, pero que adquieren valor como medios para lograr determinados fines. Pueden ser considerados en esta categoría todos aquellos bienes que sobrepasan lo necesario para la simple subsistencia pero que se relacionan con el comer y beber mejor, el gozar de servicios sanitarios más modernos y completos, etc. Se trata de ese tipo de bienes, según Honderich, que gozan las personas de los países desarrollados pero de los cuales carecen, por lo general, los que habitan en los países subdesarrollados.

3. El respeto y el autorrespeto forman la tercera categoría. Las personas desean alcanzar esta categoría como individuos y como grupo y persiguen su reconocimiento en todos los ámbitos de su actividad. Ya Rawls ha subrayado su relevancia al señalar que el autorrespeto es, con toda probabilidad, el bien primario de las personas más importante. Esta categoría incluye el sentimiento que una persona tiene de su propio valor, de su convicción acerca de que su propio plan de vida merece la pena de ser realizado. Incluye también, por cierto, la confianza que se tiene en la propia capacidad para ejecutar las personales preferencias e intenciones. "Cuando creemos que nuestros proyectos son de poco valor no podemos proseguirlos con placer ni disfrutar con su ejecución. Atormentados por el fracaso y la falta de confianza en nosotros mismos, tampoco podemos llevar adelante nuestros esfuerzos. Esta claro -continúa Rawls- por qué el auto respeto es un bien primario»» ${ }^{9}$. Es importante, naturalmente, que los hombres aprueben, confirmen y disfruten de los planes de vida de los demás, puesto que la satisfacción de esta categoría depende, en buena parte, de las respuestas que obtengamos de nuestros congéneres.

4. La cuarta categoría hace referencia a la libertad y al poder en general. Esta categoría no debe entenderse en el sentido usual y restringido de libertades políticas o de participación en la toma de decisiones políticas de la sociedad en la que uno vive,

${ }^{9}$ Cfr. John Rawls, Teoría de la Justicia, versión castellana, María Dolores González, F. C. E., 1979, pág. 486. 
sino que debe extenderse a otros ámbitos más reducidos de nuestra vida cotidiana. El nivel de vida de una persona también puede ser medido en atención a la independencia que goza en su trabajo, en su entorno familiar, etc.

5. Las personas tienen una serie de necesidades, proyectos y sentimientos que tienen que ver con su propia familia. Pero también se relacionan con otras personas con las cuales tienen, en algunos casos, una conexión lejana, a pesar de lo cual participan de sus propias esperanzas, soportes, protección, ilusión, etc. Que los hombres y mujeres establecen relaciones personales estrechas y se asocian en grupos numerosos es un hecho corriente en nuestras sociedades. Que una persona pueda hacerlo, según lo incluya o no en su propio plan de vida, constituye un dato relevante para su calidad de vida.

6. La sexta categoría está vinculada a los bienes de la cultura. No creo necesario señalar la importancia de estos bienes y su relevancia para una vida de excelencias. Es obvio que las personas persiguen afanosamente no sólo el conocimiento del mundo y el de su entorno, sino que participan también de otras experiencias como el arte, la diversión el deporte o la religión. Nadie, dice Honderich ${ }^{10}$ «elige ser incompetente o ignorante».

Ahora bien, considerar al bienestar como la satisfacción del nivel de vida tal como ha sido presentado tiene la ventaja de no hacer depender el bienestar de una persona de elementos puramente subjetivos o emocionales, a la vez que permite que se fijen cursos de acción claros con respecto a cada una de las categorías mencionadas. El artículo 50 de la Constitución Española y el artículo 31 de la Constitución de la Confederación Suiza al imponer al Estado la promoción del bienestar general parecería que hacen referencia a la promoción del nivel de vida.

$\mathrm{Si}$ en el caso anterior, donde se interpretaba el bienestar como satisfacción de deseos, se advertía contra el peligro de una actividad excesiva del Estado y se propugnaba la no intervención del mismo con la finalidad de evitar que se incurra en el perfeccionismo moral; en el caso de la interpretación del bienestar como nivel de vida se exige precisamente lo contrario. El Estado podría pecar por defecto o inacción, cuando lo éticamente exigible es su intervención no sólo para promover, sino para asegurar y garantizar a todos los ciudadanos el disfrute de los bienes que determinan los niveles de una calidad de vida aceptable. Si un Estado hiciera dejación de estas funciones, o implementara políticas contrarias a la satisfacción por todos de un

${ }^{10}$ Cfr. Ted Honderich, obra citada, pág. 483. 
nivel de vida aceptable perdería su legitimidad. En qué medida y en qué casos ha de intervenir el Estado para no hacer dejación de sus funciones y perder legitimidad por defecto, ni incurrir en un perfeccionismo moral no justificado y pecar por exceso es algo a lo que me referiré a continuación.

III

Comencé mi trabajo haciendo referencia al hecho de que en las constituciones democráticas se establece como uno de los deberes básicos del Estado la promoción, afianzamiento y garantía en su caso del bienestar de los ciudadanos, sean considerados éstos en forma individual o colectiva. Analicé después las dos versiones que suelen darse al interpretar el término bienestar: como felicidad y como nivel de vida. Se pudo observar entonces que la categoría del bienestar, en cualquiera de sus dos interpretaciones era relevante para la consideración de la legitimidad del Estado.

Esto es así, porque las personas aspiran a realizar sus propios planes de vida lo que supone que los seres humanos son capaces de formularlos autónomamente, y ya en la base misma de ambas versiones del bienestar subyace la convicción de que la autonomía de las personas constituye un elemento básico de la moral y cuyo respeto por parte del Estado ha de ser estricto si quiere mantener con éxito su pretensión de legitimidad.

Al examinar el concepto de bienestar como felicidad señalé que un Estado moderno no podía, por razones puramente empíricas, conocer todos los deseos de sus ciudadanos. Este hecho, sumado a la escasez relativa de recursos y a la imposibilidad de arbitrar soluciones frente a deseos contrapuestos podía llevar al Estado a implementar medidas de corte perfeccionista, prescindiendo de las interpretaciones individuales de cada cual. Por perfeccionismos entiendo, siguiendo a Carlos Nino, aquella concepción que afirma que lo que es bueno para una persona o lo que satisface mejor sus intereses es independiente de sus propios deseos y que el Estado puede imponer, mediante el uso de la fuerza si fuere necesario, los bienes y planes de vida que a su juicio son objetivamente mejores ${ }^{11}$. El Estado se convertiría de ese modo en árbitro de las preferencias, intereses, formas de vida e ideales humanos ${ }^{12}$.

${ }^{11}$ Cfr. Carlos S. Nino, Ética y derechos humanos, Ariel, 1989, pág. 205.

${ }^{12}$ Idem, pág. 208. 
Este podría ser incluso el caso de un Estado benevolente que persiguiera la felicidad de sus ciudadanos administrándoles medicamentos; o como en el ejemplo de la máquina de la experiencia de R. Nozlek, conectara el cerebro de sus ciudadanos a los electrodos de una máquina programada para suministrar las sensación de satisfacción de todos los deseos que posean. La máquina no dejaría secuelas y la persona a ella conectada no tendría consciencia de su dependencia. Es mas, una vez desligada de semejante vínculo tendría el sentimiento de que efectivamente vivió las experiencias deseadas.

¿Existirían razones, se pregunta Nozick, para rechazar una vida plena de satisfacciones placenteras a cambio del pequeño inconveniente de depender de una máquina? ¿Existen razones para evitar que el Estado implemente medidas de esa naturaleza? Nozick piensa que sí; y ello en virtud de que a las personas les preocupa hacer ciertas cosas; esto es, no sólo gozarlas, sino decidirlas, elaborarlas y ejecutarlas por sí mismas. Pero no únicamente desean hacer cosas, sino que quieren ser de determinada manera. No desean ser un simple instrumento fabricado por una máquina o modelado por el Estado. Las personas quieren ser, en definitiva, seres autónomos ${ }^{13}$.

Por eso tiene razón Griffin cuando afirma que en importantes áreas de la vida, las personas prefieren conocer la amarga verdad antes que vivir confortablemente engañados. Y aún en la hipótesis de si «estuviera rodeado por consumados actores capaces de proporcionarme dulces simulacros de cariño y amor, yo preferiría la relativamente amarga dieta de sus reacciones auténticas. Y lo preferiría no porque fuera moralmente mejor o estéticamente mejor o más noble, sino porque contribuiría a hacer una mejor vida para mí, más digna de ser vivida» ${ }^{14}$.

Si este núcleo central de la moral que es la autonomía de las personas -repitoquedase afectada por cualquier medida del Estado que aún con finalidad benevolente impusiera criterios perfeccionistas de moralidad provocaría que su pretensión de legitimidad quedara severamente afectada.

Con respecto a los niveles de bienestar entendidos ya desde el punto de vista objetivo y su distribución en la sociedad Ted Honderich ha propuesto el llamado principio de igualdad que pienso es interesante recordar aquí. Según este principio se debería dar prioridad a las medidas políticas, cuyos fines sean hacer

${ }^{13}$ Cfr. Robert Nozick, Anarquía, Estado y Utopía, versión castellana, Rolando Tamayo, Fondo de Cultura Económica, 1988, págs. 53 y sigs.

${ }^{14}$ Cfr. James Griffin, obra citada, pág. 9. 
que estén mejor aquellos que están peor transfiriendo medios de aquellos que están mejor a los que están peor. Se afectará primero a aquellos que tienen el nivel más alto y se observará como límite la reducción de las desigualdades necesarias.

Por cierto que la aplicación de este principio merece algunas aclaraciones previas. En primer lugar, subrayar que la aplicación de este principio no está vinculada únicamente a la distribución de bienes materiales, sino que afecta a todos los aspectos del bienestar. La expresión «los que están peor», por tanto, no se refiere exclusivamente a quienes carecen de bienes necesarios para la subsistencia, sino que hace referencia a quienes les está vedado el disfrute del conjunto de las categorías mencionadas. Nada dice en cambio sobre aquellas personas que habiendo satisfecho las exigencias de una categoría se encuentran peor situadas que otras respecto a esa misma categoría.

En segundo lugar, este principio no persigue un igualitarismo absoluto. De lo que se trata aquí es de desplazar una parte de los recursos de los que están mejor situados en una cantidad tal que tienda a reducir las desigualdades necesarias ${ }^{15}$.

Desde una perspectiva objetiva, el bienestar de una persona depende, en primer lugar, de la satisfacción de las necesidades básicas primarias o derivadas. Ninguna otra política o programa de gobierno puede ser prioritaria respecto de ese objetivo. No se tratará aquí naturalmente de implementar medidas informadas por el principio de caridad, se trata más bien de realizar actos de justicia. Las personas que sufren hambre, enfermedad o extrema pobreza no deben implorar pidiendo ayuda. Ellas deben exigirla, y el Estado proveerla $^{16}$.

El Estado debería garantizar, a riesgo de perder su legitimidad, lo que Ernesto Garzón denominó el «coto vedado» de las personas ${ }^{17}$. En este sentido, el concepto de bienestar como nivel de vida sí que se manifiesta interesante para valorar la justicia o injusticia de una sociedad. La referencia al «coto vedado» tiene relevancia para la satisfacción de las categorías mencionadas por Honderich, ya que dicho «coto» incluye tanto la satisfacción de necesidades básicas primarias y derivadas, como el respeto del principio de igualdad.

Y del concepto de bienestar entendido como felicidad qué quedaría. Asumir tal concepción es importante porque en una

\footnotetext{
${ }^{15} \mathrm{Cfr}$. Ted Honderich, obra citada, págs. 484 y sigs.

${ }^{16}$ Cfr. John Baker, Arguing for Equality, Verso -New York- 1987, págs. 17 y sigs.

${ }^{17}$ Cfr. Ernesto Garzón Valdés, «Representación y democracia», en Doxa, núm. 6, 1989, págs. 143 y sig.
} 
sociedad democrática las distintas fuerzas políticas están interesadas en satisfacer los deseos secundarios de las personas por medio de la negociación y del compromiso. La persecución de la felicidad a la que hacía referencia Jefferson en la primera versión de la Declaración de Independencia de los Estados Unidos o la Constitución de Cádiz recobran así toda su fuerza y su sentido si se entiende que en una democracia el Estado debe suministrar un marco adecuado para que los ciudadanos hagan valer también su concepción de la felicidad.

En resumen, el concepto de bienestar tiene relevancia tanto si se lo interpreta en el sentido de la defensa del «coto vedado» o de las categorías propuestas por Honderich; como si se lo interpreta bajo el prisma de la satisfacción de los deseos secundarios. En su versión objetiva, el concepto de bienestar está muy vinculado al concepto de necesidades básicas y su realización requiere el cumplimiento por parte del Estado no sólo de deberes negativos, sino también positivos. En su versión subjetiva, el bienestar apunta a la realización de los deseos secundarios de las personas y por ello choca frontalmente contra toda ideología perfeccionista.

Tanto en la versión subjetiva como en la objetiva a lo que se aspira es a una mayor protección de la autonomía de las personas; es decir, tiende a apuntalar uno de los valores básicos de una concepción democrática y liberal de la sociedad.

Vistas así las cosas, el concepto de bienestar tiene relevancia como elemento que nos permite juzgar acerca de la legitimidad de los ordenamientos jurídico-políticos. Esta relevancia es lo que explica en mi opinión su inclusión en documentos constitucionales y en declaraciones básicas como las mencionadas al comienzo de este trabajo. 